\title{
Design and Fabrication of a Novel 3D Micropellistor
}

\author{
Lei Xu, Tie Li, Xiuli Gao, Yuelin Wang \\ State Key Laboratory of Transducer Technology, Science and Technology on Micro-system \\ Laboratory, Shanghai Institute of Microsystem and Information Technology, Chinese Academy of \\ Sciences, Shanghai, China \\ tli@mail.sim.ac.cn
}

\begin{abstract}
:
This paper presents a novel 3D micropellistor based on a 3D microheater. The 3D microheater with a $\mathrm{Pt}$ heater embedded in a concave-shaped active area was fabricated by MEMS technology. Then a detector and a compensator was developed by sol-gel process, introducing Pd as the catalytic metal and porous alumna as the reference material. Test results indicate that the 3D catalytic micropellistor has a output voltage of $12 \mathrm{mV}$ to $10 \%$ LEL methane. And it has a very high sensitivity, fast response time and liner output voltages.
\end{abstract}

Key words: micropellistor, 3D microheater, MEMS, and sol-gel

\begin{abstract}
Introduction
Catalytic pellistor gas sensors have been widely used for detecting combustible gases and vapours in air for several decades [1]. Due to the low power, low cost, and potential of batch production, micro-heater based gas sensors have been extensively studied for more than two decades. Current micro-heater based micropellistor gas sensors often have lower sensitivity in comparison with that of conventional sensors. The sensitivity of the conventional sensors is about $15-30 \mathrm{mV} / \% \mathrm{CH}_{4}$ calibrated by $50 \% \mathrm{LEL} \mathrm{CH}_{4}$. Some micro-heater based pellistor gas sensors reported in recent papers have a sensitivity of about $5 \mathrm{mV} / \% \mathrm{CH}_{4}$ $[2,3]$. It is mainly because sensing materials loaded on the active area are less than those of the conventional sensors, generating less heat during combustion reaction.
\end{abstract}

Our previous paper presented a novel 3D micro-heater which has a concave-shaped active membrane [4]. The unique structure makes it convenient to load more catalytic materials into the active area. It also makes a better utilization of heat, which may therefore improve the sensitivity. This paper reports on a pellistor gas sensor based on the 3D microheater. The detailed design, fabrication, and testing results will be given in the following sections.

\section{Sensing Mechanism}

Pellistor gas sensors detect flammable gases by monitoring resistance change of the $\mathrm{Pt}$ heater resulting from temperature increase produced by combustion heat. The detecting mechanism can be divided into four sub-steps, shown in Fig. 1. In the first step, combustible gases (typically methane) mixed with air burn and induce heat with the help of catalyst, which makes chemical energy transform into thermal energy. Secondly, a part of the combustion heat loss to the environment through conduction, convection, and radiation, with another part of the combustion heat transfers into Pt heater and causes a temperature increase $(\Delta T)$ of the $P t$ heater. Thirdly, due to the nature of platinum, resistance of $\mathrm{Pt}$ heater increases $(\Delta \mathrm{R})$ with the increasing of temperature $(\Delta T)$. Finally, the flammable gases can be detected by measuring the voltage change which results from the resistance increase $(\Delta R)$ with a Wheatstone bridge circuit.

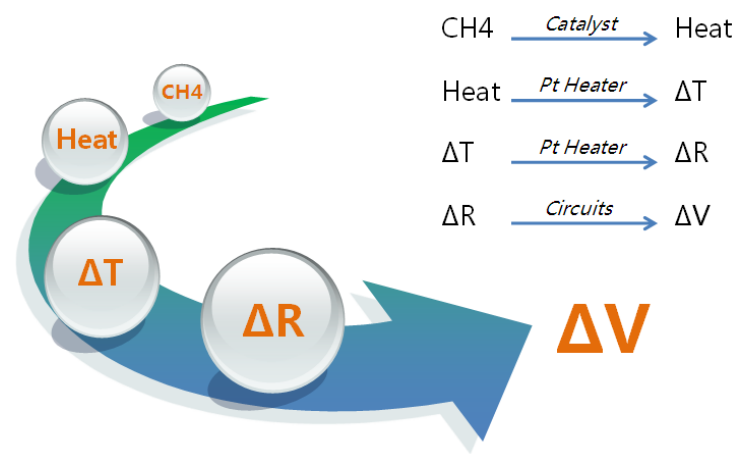

Fig. 1. Sensing mechanism of pellistor gas sensors.

From the four steps of detecting mechanism described above, sensitivity can by effectively improved by structure optimization through two 
ways. One is using micro-heaters with larger active area which can load more catalyst and generate more heat during oxidation. Another way is to improve the utilization of combustion heat, making more heat isolated in the active area by reducing heat losses to environment.

\section{Structure Design}

We designed a novel 3D micro-heater for micropellistor gas sensors to improve the performance. Fig. 2 shows the structure of the 3D micro-heater. The structure is quite simple. A concave-shaped membrane with a Pt heater embedded in it is supported by two beams. For thermal isolation, the whole structure is suspended over a silicon pit.

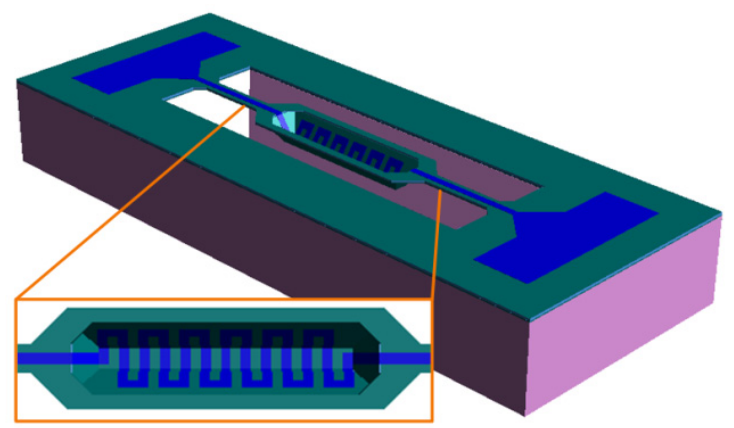

Fig. 2. Model of the 3D micro-heater.

This 3D micro-heater with a concave shaped membrane has higher heating efficiency than current 2D micro-heaters [4]. Catalyst and porous alumina can be synthesized right into the concave active area by sol-gel process. The size-increased active area with more catalyst loaded can generate more heat during combustion reaction. And the concave-shaped structure can help increase the utilization of combustion heat by reducing heat loss via convection and radiation.

\section{Micro-heater Fabrication}

The 3D micro-heater was fabricated by MEMS technology. The fabrication process steps are shown in Fig. 3 and detailed as follows.

(a) The fabrication process started from a 4inch n-type (100) silicon wafer with a resistivity of $1-10 \Omega \cdot \mathrm{cm}$. Then a layer of $300 \mathrm{~nm}$-thick silicon oxide was thermally grown on the front side and back side of the wafer.

(b) A rectangular opening with the four right angles cut was formed by RIE. The four sides of the rectangular opening are $\mathrm{Si}$ $<110>$ oriented.

(c) Then, the first anisotropic etching was processed. A concave cavity with the depth as 30-40 $\mu \mathrm{m}$ was formed by $\mathrm{KOH}$ etching through the rectangular etching opening.

(d) Then a concave-shaped dielectric membrane was formed by deposition of a layer of $300 \mathrm{~nm}$-thick silicon oxide and a layer of $1000 \mathrm{~nm}$-thick low-stress silicon nitride. Due to the four missing right angles of the rectangular opening, four suspended triangle membranes was also formed to enhance the robustness of the concave shaped membrane.

(e) Using a commercial spray-coating machine EVG-101, a layer of $4 \mu \mathrm{m}$-thick photoresist was spray-coated on the surface of both the concave dielectric membrane bottom/sidewalls and the top dielectric membrane on the substrate. Then a $200 \mathrm{~nm}$-thick Pt heating resistor and bonding pads with adhesive layer buried beneath Pt were formed by lift-off process.

(f) The stacked membrane of $\mathrm{SiO}_{2} / \mathrm{SiN}_{\mathrm{x}}$ was etched by RIE to define the active area and suspension beams with two etching openings formed at the same time.

(g) Then the silicon substrate was etched by DRIE with a depth as about $25 \mu \mathrm{m}$ so as the two supporting beams and the concave-shaped membrane can be released by wet chemical anisotropic etching.

(h) Finally, the second anisotropic etching was processed. The whole structure was released using a tetramethylammonium hydroxide (TMAH) solution at $80^{\circ} \mathrm{C}$.

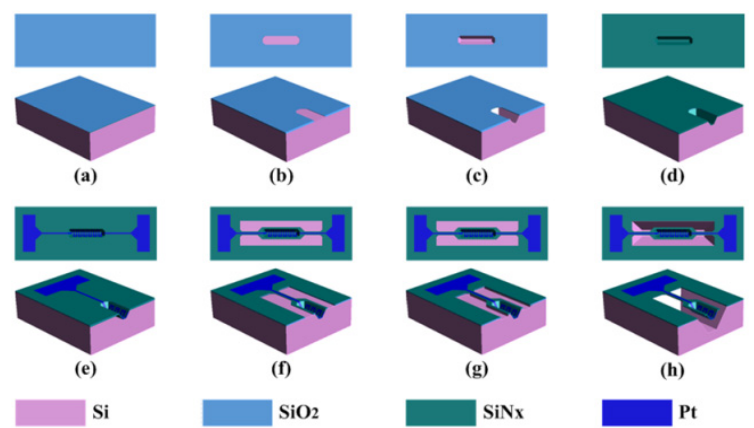

Fig. 3. Fabrication process of the 3D micro-heater.

\section{Sensor Fabrication}

After the MEMS process ,a micropellistor gas sensor was developed by sol-gel process. By introducing $\mathrm{y}-\mathrm{Al}_{2} \mathrm{O}_{3}$ supported $15 \mathrm{wt} \% \mathrm{Pd}$ as catalyst, alumina aerogel and palladium chloric 
acid mixed with alumina aerogel were separately drop coated into the concave membranes of the 3D micro-heaters. After electrical heating at $600^{\circ} \mathrm{C}$ for $5 \mathrm{~min}$, the alumina aerogel was sintered into meso-porous $\mathrm{Y}-\mathrm{Al}_{2} \mathrm{O}_{3}$ support, while high activity palladium particles were formed at the same time (see Fig. 4).

The micro-heater with $\mathrm{y}-\mathrm{Al}_{2} \mathrm{O}_{3}$ supported $15 \mathrm{wt}$ $\% \mathrm{Pd}$ acts as a detector to promote combustion of combustible gases. While the micro-heater with only porous $\mathrm{Y}-\mathrm{Al}_{2} \mathrm{O}_{3}$ loaded acts as a compensator to prevent such combustion and to decrease the influence of temperature and humidity.

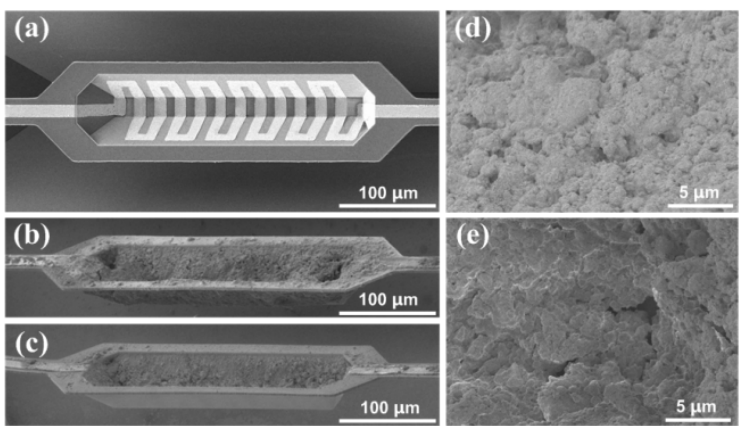

Fig. 4. SEM images of the $3 D$ micro-heater (a), detector (b), compensator (c), Pd catalyst (d), porous $\mathrm{Al}_{2} \mathrm{O}_{3}(d)$

\section{Temperature Versus Power}

Electrical characteristics of the 3D micro-heater and sensor have been tested. Fig. 5 shows the temperature versus power curve for the 3D micro-heater, compensator and detector. The temperature increases with the increasing of power consumption. It is observed from the curve that there is a slight temperature shift of the detector and compensator in comparison with the bare 3D micro-heater. When heated to $400^{\circ} \mathrm{C}$, the bare 3D micro-heater only consumed about $20 \mathrm{~mW}$. While it increased to $25 \mathrm{~mW}$ for the detector and compensator. That is because heat losses via convection and conduction should increase, when catalyst and $\mathrm{Y}-\mathrm{Al}_{2} \mathrm{O}_{3}$ loaded.

The total power for the micropellistor gas sensor with paired detector and compensator is $50 \mathrm{~mW}$, which is lower than commercially available traditional LEL sensors with a typical static power consumption of $250 \mathrm{~mW}$.

From the inlet of Fig. 5, power per active area (PPAA) of the 3D micro-heater is only half or less than current well designed 2D microheaters [5-7]. The 3D micro-heater solves the dilemma in power consumption and sensitivity. It has a large concave-shaped active area for loading catalyst with a remarkable high heating efficiency. And the occupation of chip area is as large as 2D micro-heaters.

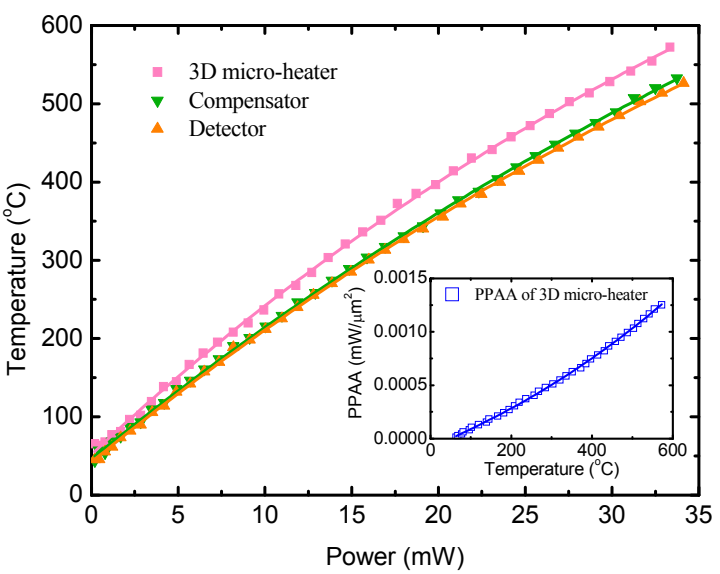

Fig. 5. Temperature versus power of the $3 D$ microheater, compensator, and detector.

\section{Test System}

A bridge circuit shown in Fig. 6 was used to get output signal of the micropellistor gas sensors. It is composed of a detector (sensing element), a compensator (reference element), and two fixed resistors. And this circuit does not have any amplifiers, filters, or signal process circuits. So the output $\left(\Delta V=V_{1}-V_{2}\right)$ is the original signal. Sensitivity of the gas sensor is defined as the output voltage per every percentage of methane $\left(\mathrm{mV} / \% \mathrm{CH}_{4}\right)$.

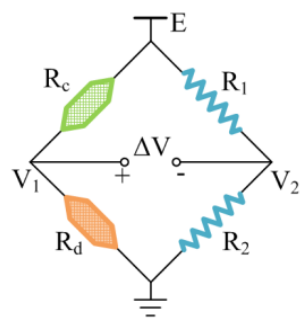

Fig. 6. Bridge circuits for testing. $R_{d}$ : detector, $R_{c}$ : compensator, $R_{1}, R_{2}$ : resistors, $\Delta V=V_{1}-V_{2}$.

Methane, which is a typical flammable gas and should be especially monitored in the mining industry, was selected as the target gas to detect, so as to evaluate the performance of the $3 \mathrm{D}$ micropellistor gas sensor.

\section{Sensor Response to Methane}

By applying an electric power, the sensors were operated at temperature of $400^{\circ} \mathrm{C}$. Fig. 7 (a) shows the original signal of the sensor response to methane with different concentrations. The gas sensor was sensitive to $10 \% \mathrm{LEL} \mathrm{CH}_{4}$ (the first alarm level) with a output voltage as $12 \mathrm{mV}$. The sensitivity to $50 \%$ LEL $\mathrm{CH}_{4}$ was about $12 \mathrm{mV} / \% \mathrm{CH}_{4}$ with a response time $\left(T_{90}\right)$ less than $10 \mathrm{~s}$. Fig. 7 (b) Shows the linear fit of outvoltage to menthane 
concentration. Output voltage has a good linear relation to methane concentration.

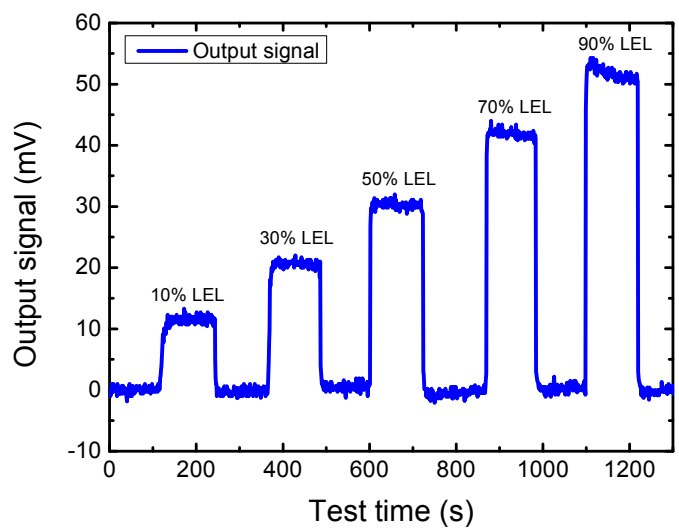

(a)

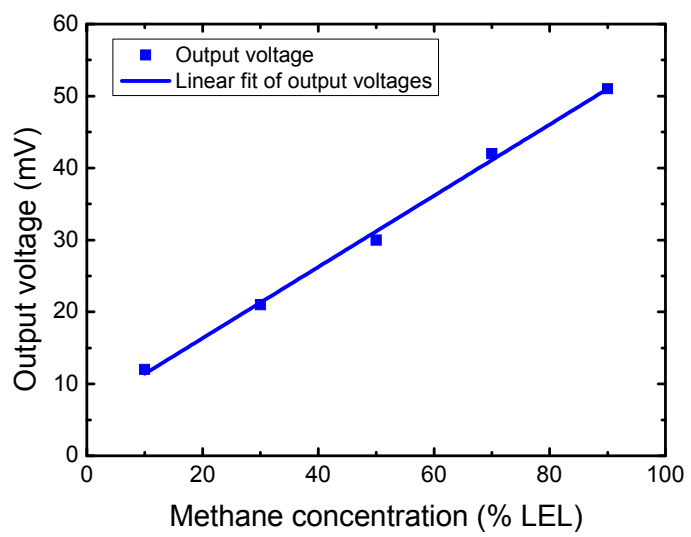

(b)

Fig. 7. Sensor response to methane. (a) the original output signal to methane from $10 \%$ LEL to $90 \%$ LEL, (b) linear fit of output voltage to methane concentration.

Sensitivity of this micropellistor gas sensor is higher than some micropellistor gas sensors based on 2D micro-heaters. And it is alomst as high as the convensitional sensors.

As mentioned before, there are two advantages of the 3D micro-heater that can help improve the sensitivity. On one hand, the actual active area of the 3D micro-heater is larger than that of common 2D micro-heaters. More combustion heat was released inside the concave membrane. On the other, due to the unique capability of concentrating heat [4], the 3D micro-heater made better use of the combustion heat over the 2D micro-heater, which further improves the sensitivity.

\section{Conclusion}

A novel 3D micro-heater and its application in pellistor gas sensors have been presented. The 3D micro-heater has been fabricated using classical MEMS technology with carefully designed processes. It has extremely high heating efficiency in comparison with that of current 2D micro-heaters. By introducing $\mathrm{Y}$ $\mathrm{Al}_{2} \mathrm{O}_{3}$ supported 15 wt $\% \mathrm{Pd}$ as sensing material, a 3D micropellistor gas sensor has been achieved based on the 3D micro-heaters with low power consumption, fast response time, and high sensitivity.

\section{Acknowledgements}

This work was supported in part by the National Basic Research Program of China under Grant 2011CB309501, by the National Basic Research Program of China under Grant 2012CB934102, by the National Hi-tech Research and Development Program of China under Grant SS2012AA040402, by the Fund for Creative Research of National Natural Science Foundation of China (NSFC) under Grant 61021064 , by the Key project of NSFC under Grants 60936001 and 91123037.

\section{References}

[1] A. Hierlemann, O. Brand, C. Hagleitner, and H. Baltes, Microfabrication techniques for chemical/biosensors, Proceedings of the IEEE, 91, 839-863, (2003); doi: 10.1109/JPROC.2003.813583

[2] W. Shin, K. Tajima, Y. S. Choi, N. Izu, I. Matsubara, and N. Murayama, Planar catalytic combustor film for thermoelectric hydrogen sensor, Sensors and Actuators B-Chemical, 108, 455-460, (2005); dio: 10.1016/j.snb.2004.12.077

[3] L. Xu, T. Li, X. L. Gao, Y. L. Wang, R. Zheng, L. Xie, and L. C. Lee, Behaviour of a Catalytic Combustion Methane Gas Sensor Working on Pulse Mode, 2010 IEEE Sensors, 391-394, (2010); dio: 10.1109/ICSENS.2010.5690213

[4] L. Xu, T. Li, and Y. L. Wang, A novel threedimensional microheater, IEEE Electron Device Letters, 32, 1284-1286, (2011); doi: 10.1109/LED.2011.2160145

[5] J. Lee, C. M. Spadaccini, E. V. Mukerjee, and W. P. King, Differential Scanning Calorimeter Based on Suspended Membrane Single Crystal Silicon Microhotplate, Journal of Microelectromechanical Systems, 17, 1513-1525, (2008); doi: 10.1109/JMEMS.2008.2006811

[6] L. Xu, T. Li, X. L. Gao, and Y. L. Wang, Development of a Reliable Micro-Hotplate With Low Power Consumption, IEEE Sensors Journal, 11, 913-919, (2011); doi: 10.1109/JSEN.2010.2064765

[7] I. Elmi, S. Zampolli, E. Cozzani, F. Mancarella, and G. C. Cardinali, Development of ultra-lowpower consumption MOX sensors with ppb-level VOC detection capabilities for emerging applications, Sensors and Actuators B-Chemical, 135, 342-351, (2008); doi: 10.1016/j.snb.2008.09.002 